\title{
Stochastic Analysis of Two Non-identical Unit Parallel System Incorporating Waiting Time and Preventive Maintenance
}

\author{
S. E. Abo-Youssef ${ }^{1}$, Fatima A. Assed ${ }^{2}$ \\ Mathematics Department, Faculty of Science, Al-Azhar university, Nasr City (11884), Cairo, Egypt ${ }^{1}$ \\ Mathematics Department, Faculty of Science, Helwan university, Helwan, Egypt ${ }^{2}$ \\ abuyoussed@azhar.edu.eg ${ }^{1}$,ftm942444@gmail.com²
}

\begin{abstract}
The reliability of two non-identical unit's parallel system with two kinds of failures common cause failure and partial failures is inspected. Moreover, the preventive maintenance and waiting time to repair, a significant aspect of reliability analysis, has also been incorporated. The proposed system is assumed to a function properly if at least one of the unit is in operate mode. The system goes for preventive maintenance at random apaches. Supplementary variable technique and Laplace transform have used for solution. Our results are compared with the previous results to observe the effect of preventive maintenance and failure rates on system performance.
\end{abstract}

Key words: Reliability, Steady-State Availability, Mean Time to System Failure (MTTF), Preventive Maintenance, Parallel System.

Date of Publication: 2018-10-10

DOI: https://doi.org/10.24297/jam.v14i2.7763

ISSN: 2347-1921

Volume: 14 Issue: 02

Journal: Journal of Advances in Mathematics

Website: https://cirworld.com

This work is licensed under a Creative Commons Attribution 4.0 International License. 


\section{Introduction}

Reliability analysis plays key role in designing of advanced systems such as nuclear weapons control systems, power supply systems, software systems, satellite control systems, telecommunication systems. In today's competitive world, it has become important to produce the cost-effective, efficient, user-friendly systems along with the criteria of reliability. The complex systems with different failure and repair polices have been considered by earlier researchers like Gupta and Agarwal (1984), Dhillon and Yang (1992a, 1992 b, 1993) and Gupta and Sharma (1993). Reliability analysis of parallel systems has been extensively studied by Dhillon and Viswanath (1991), Kumar et al. (2012) and Agnihotri and Satsangi (1996).

The common cause failure analysis of a two non-identical unit parallel system with arbitrarily distributed repair times has been studied by Dhillon and Anuda (1993a). Sridharan and Mohanavadivu (1997) studied the reliability measures such as availability, steady state system availability and mean time to failure (MTTF) for two nonidentical unit parallel system with two types of failure, namely, common cause and human error.

Agnihotri and Satsangi (1996) computed the reliability of the same system but with the priority of base repair and inspection by using the regenerative point technique. Mohamed and Sherbeny (2013) investigated a stochastic analysis of two dissimilar unit parallel system with common cause failure, critical human error, noncritical human error and preventive maintenance. Kadyan et al. (2004), Chander (2005) and Malik et al. (2010) are introduced some further studies focusing on the reliability of non-identical unit parallel system.

An analytical approach to compute the reliability of the system which contains mixed configuration has been presented by Ram and Kumar (2015). Ram and Kumar (2015) have emphasized on the importance of repair facility by showing that the availability is much higher with respect to reliability for the same time unit. It is a well-known fact that the waiting time to repair is an important aspect of reliability analysis.

Ram et al. (2013) investigated the reliability of a standby system incorporating a waiting time to repair. Singh and Gulati (2014) considered a system where the main unit and a standby unit are assumed to go under two types of failures: partial failure and complete failure. Singh and Gulati (2014) assumed that if the repair facility is not available, the system will have to wait for repair until the repair facility is available.

Ram and Manglik (2014) studied a complex repairable system having three units in parallel configuration with three types of failures, namely, partial failure, catastrophic failure and human failure. In the case of partial failure, the system waits for repair and when there is no repairman to repair, it goes to the complete failure mode. It is obvious that the use of identical components increases cost, complexity and time to design. The high cost may be reduced by using non-identical units in parallel. Home lightning circuit consisting of two non-identical lamps is an example of such system. The main advantage of the parallel connection is that if one of the lamp burns out then there is no effect on the second one and it keeps operating.

Preventive maintenance refers to regular, routine maintenance to help keep units up and running, preventing any unplanned downtime and expensive costs from unanticipated unite failure. It requires careful planning and scheduling of maintenance on unit before there is an actual problem as well as keeping accurate records of past inspections and servicing reports. Preventive maintenance involves the systematic inspection of units where potential problems are detected and corrected in order to prevent unite failure before it happens. In practice, a preventive maintenance schedule may include things such as cleaning, lubrication, oil changes, adjustments, repairs, inspecting and replacing parts, and partial or complete overhauls that are regularly scheduled. Therefore, Systems with preventive maintenance are more likely to have greater MTTF and their availability is also greater that those systems without preventive maintenance. This is our main focus for the proposed system. Comparison of reliability characteristics of two systems with preventive maintenance and different modes by El-Said, K. M., \& El-hamid, R. A. (2008).

Chopra and Ram (2017) evaluated various reliability of two non-identical unit parallel system with types of failures common cause of failure and partial failure incorporating waiting time. In this paper, we investigate 
the reliability of two non-identical unit's parallel system with two kinds of failures common cause and partial of failures incorporating preventive maintenance and waiting time to repair. The system goes for preventive maintenance at random apaches. Supplementary variable technique and Laplace transform have used for solution.

Our results are compared with the previous results to observe the effect of preventive maintenance and failure rates on system performance.

\section{Nomenclature and system states}

The present system consists of two non-identical units: A and B in parallel configuration.

$t:$

$S:$

$s_{i}=(i=0,1,2,3,4,5,6,7):$

$\lambda_{a} \backslash \lambda_{b}:$

$\lambda_{c c} \backslash \lambda_{c c a} \backslash \lambda_{c c b}:$

$\lambda_{P} \backslash \lambda_{P a} \backslash \lambda_{P b}:$

$w:$

$\mu_{3}(x) \backslash \mu_{5}(x) \backslash \mu_{6}(x):$

$P_{i}(t):$

$\overline{P_{l}(s)} \quad$ :

$P_{3}(x, t) P_{5}(x, t) P_{6}(x, t):$

$\overline{q_{3}(s)} \backslash \overline{q_{5}(s)} \backslash \overline{q_{6}(s)}:$

$\theta_{1}:$

$\theta_{2}:$

$A(t):$

$R(t)$ :

MTTF:

Laplace transform of $P_{i}(t)$ is:
Time scale.

Laplace transform Variable.

Transition states for $(i=0,1,2,3,4,5,6,7)$

Failure rate of units $A$ and $B$.

Rate of common cause Failure when both Units working/unit A Failed/unit $B$ failed.

Rate of partial failure When both units Working/unit A Failed/unit B failed.

Waiting time to repair

Repair rate for failed states $s_{3} \backslash s_{5} \backslash s_{6}$.

Probability that the System is in $s_{i}=(i=0,1,2,3,4,5,6,7)$ state at Time $t$

Laplace transform of $P_{i}(t)$

Probability density function that the system is in state $s_{3} \backslash s_{5} \backslash s_{6}$ At time t and has an elapsed repair time $x$

Laplace transform of probability density functions of repair Rates $\mu_{3}(x) \backslash \mu_{5}(x) \backslash \mu_{6}(x)$.

Rate of time for taking a unit into preventive maintenance.

Rate of preventive maintenance time.

Function of availability.

Function of reliability.

Mean time to failure.

$\overline{P_{l}(s)}=\int_{0}^{\infty} e^{-s t} P_{i}(t) d t$ 


\section{Model description and assumptions}

In this section, we apply preventive maintenance to a system consisting of two non-identical units in parallel configuration with two types of failures: common cause failure and Partial failure. The system will be in operative mode if at least one of the unite is functioning property. A common cause failure is defined as any instance where multiple unites fail due to a single cause (Dhillon and Viswanath, 1991). The main causes of common cause failure are designing deficiencies, operations and maintenance errors, external catastrophe and functional deficiency. The partial failure is caused in the system due to partial failure of internal unites. The different states of the system are given in the following:

\section{The state description}

$s_{0}$ : Good state as both units are working normally.

$\boldsymbol{s}_{1}$ : Partially failed state as unit A has failed and unit B is operative.

$s_{2}$ : Partially failed state as unit B has failed and unit A is functional.

$s_{3}$ : Completely failed state due to failure of both units.

$s_{4}$ : Partially failed state due to partial failure.

$s_{5}$ : Completely failed state because of the unavailability of repairman.

$s_{6}$ : Completely failed state due to common cause failure.

$s_{7}$ : Two units under preventive maintenance.

\section{The system:}

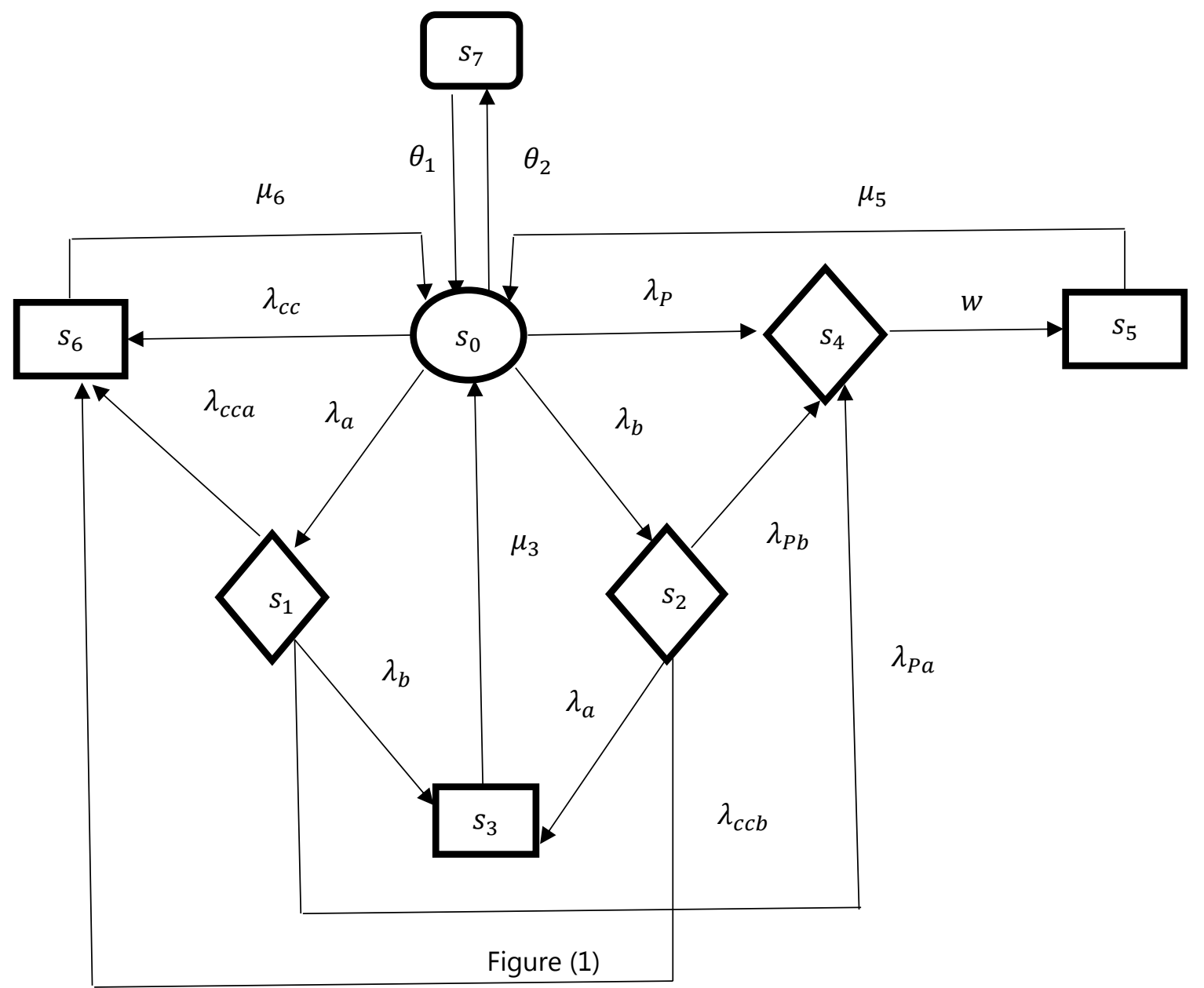


The following assumptions are associated with this mathematical model

(1) The system consists of two non-identical parallel units.

(2) Initially both units are operative, and the system is in good state.

(3) The system has three states: good, partially failed and completely failed state.

(4) The system goes to completely failed state as a result of common cause failure, unavailability of repairman and failure of both units.

(5) In the case of partial failure, system waits for repair.

(6) The common cause and other failures are statistically independent.

(7) Common cause failure and partial failure can occur when both units are functioning $\left(s_{0}\right)$; furthermore, when one unit has failed and the other is working normally $\left(s_{1} / s_{2}\right)$.

(8) All failure rates are constant, while repairs follow general distribution.

(9) The system is repaired only if it is in completely failed state.

(10) The repaired system is on a par with new one.

\section{Analysis and discussion}

The present mathematical model in figure (1) is governed by the following set of differential equations

1) $\left(\frac{\partial}{\partial t}+\lambda_{a}+\lambda_{b}+\lambda_{c c}+\lambda_{p}+\theta_{2}\right) P_{0}(t)=\int_{0}^{\infty} \mu_{3}(x) P_{3}(x, t) d x+\int_{0}^{\infty} \mu_{5}(x) P_{5}(x, t) d x+\int_{0}^{\infty} \mu_{6} P_{6}(x, t) d x+\theta_{1} P_{7}(t)$

2) $\left(\frac{\partial}{\partial t}+\lambda_{b}+\lambda_{p a}+\lambda_{c c a}\right) P_{1}(t)=\lambda_{a} P_{0}(t)$

3) $\left(\frac{\partial}{\partial t}+\lambda_{a}+\lambda_{p b}+\lambda_{c c b}\right) P_{2}(t)=\lambda_{b} P_{0}(t)$

4) $\left(\frac{\partial}{\partial t}+\frac{\partial}{\partial x}+\mu_{3}\right) P_{3}(x, t)=0$

5) $\left(\frac{\partial}{\partial t}+w\right) P_{4}(t)=\lambda_{p} P_{0}(t)+\lambda_{p a} P_{1}(t)+\lambda_{p b} P_{2}(t)$

6) $\left(\frac{\partial}{\partial t}+\frac{\partial}{\partial x}+\mu_{5}\right) P_{5}(x, t)=0$

7) $\left(\frac{\partial}{\partial t}+\frac{\partial}{\partial x}+\mu_{6}\right) P_{6}(x, t)=0$

8) $\left(\frac{\partial}{\partial t}+\theta_{1}\right) P_{7}(t)=\theta_{2} P_{0}(t)$

At time $t=0$ we have:

$P_{0}(0)=1$

$P_{1}(t)=P_{2}(0)=P_{3}(0)=P_{4}(0)=P_{5}(0)=P_{6}(0)=P_{7}(0)=0$

Boundary conditions are the following:-

$P_{3}(0, t)=\lambda_{b} P_{1}(t)+\lambda_{a} P_{2}(t)$

$P_{5}(0, t)=w P_{4}(t)$

$P_{6}(0, t)=\lambda_{c c} P_{0}(t)+\lambda_{c c a} P_{1}(t)+\lambda_{c c b} P_{2}(t)$ 


\section{Solution of the model}

By taking the Laplace transform of Equations from(4.1)to (4.8) and the boundary conditions, we get

$$
\left(s+\lambda_{a}+\lambda_{b}+\lambda_{c c}+\lambda_{p}+\theta_{2}\right) \overline{P_{0}(s)}=\int_{0}^{\infty} \mu_{3}(x) \overline{P_{3}(x, s)} d x+\int_{0}^{\infty} \mu_{5}(x) \overline{P_{5}(x, s)} d x+\int_{0}^{\infty} \mu_{6} \overline{P_{6}(x, s)} d x+\theta_{1} \overline{P_{7}(s)}
$$

$\left(s+\lambda_{b}+\lambda_{p a}+\lambda_{c c a}\right) \overline{P_{1}(s)}=\lambda_{a} \overline{P_{0}(s)}$

$\left(s+\lambda_{a}+\lambda_{p b}+\lambda_{c c b}\right) \overline{P_{2}(s)}=\lambda_{b} \overline{P_{0}(s)}$

$\left(s+\frac{\partial}{\partial x}+\mu_{3}\right) \overline{P_{3}(x, s)}=0$

$(s+w) \overline{P_{4}(s)}=\lambda_{p} \overline{P_{0}(s)}+\lambda_{p a} \overline{P_{1}(s)}+\lambda_{p b} \overline{P_{2}(s)}$

$\left(s+\frac{\partial}{\partial x}+\mu_{5}\right) \overline{P_{5}(x, s)}=0$

$\left(s+\frac{\partial}{\partial x}+\mu_{6}\right) \overline{P_{6}(x, s)}=0$

$\left(s+\theta_{1}\right) \overline{P_{7}(s)}=\theta_{2} \overline{P_{0}(s)}$,

With Boundary conditions

$$
\begin{aligned}
& \overline{P_{3}(0, s)}=\lambda_{b} \overline{P_{1}(s)}+\lambda_{a} \overline{P_{2}(s)} \\
& \overline{P_{5}(0, s)}=w \overline{P_{4}(s)} \\
& P_{6}(0, s)=\lambda_{c c} \overline{P_{0}(s)}+\lambda_{c c a} \overline{P_{1}(s)}+\lambda_{c c b} \overline{P_{2}(s)} .
\end{aligned}
$$

Now let the first integral of equation (4.14) is given by

$I=\int_{0}^{\infty} \mu_{3}(x) \overline{P_{3}(x, s)} d x$

In view of equation (4.17) we have

$\overline{P_{3}(x, s)}=\overline{P_{3}(0, s)} e^{-s x} e^{-\int_{0}^{x} \mu_{3} d u} d x$

In view of (4.25) the first integral becomes as the following

where

$$
\begin{aligned}
I & =\overline{P_{3}(0, s)} \int_{0}^{\infty} \mu_{3}(x) e^{-s x} e^{-\int_{0}^{x} \mu_{3} d u} d x \\
& =\overline{P_{3}(0, s)} q_{3}(s),
\end{aligned}
$$

$q_{3}(s)=\int_{0}^{\infty} \mu_{3}(x) e^{-s x} e^{-\int_{0}^{x} \mu_{3} d u} d x$

By using the boundary condition (4.22), the first integral of equation (4.14) becomes

$I=\left[\lambda_{b} \overline{P_{1}(s)}+\lambda_{a} \overline{P_{2}(s)}\right] \overline{q_{3}(s)}$

From equation(4.15) and (4.16), we obtain

$\overline{P_{1}(s)}=\frac{\lambda_{a}}{s+B_{1}} \overline{P_{0}(s)}$,

where $B_{1}=\lambda_{b}+\lambda_{p a}+\lambda_{c c a}$ ，

and

$\overline{P_{2}(s)}=\frac{\lambda_{b}}{s+B_{2}} \overline{P_{0}(s)}$

where: $\quad B_{2}=\lambda_{a}+\lambda_{p b}+\lambda_{c c b}$.

Using (4.27) and (4.28) in (4.26) we give

$I=\left[\lambda_{b} \frac{\lambda_{a}}{s+B_{1}}+\lambda_{a} \frac{\lambda_{b}}{s+B_{2}}\right] \overline{P_{0}(s)} \overline{q_{3}(s)}$.

Let the second integral of equation (4.14) is given by

$I I=\int_{0}^{\infty} \mu_{5}(x) \overline{P_{5}(x, s)} d x$ 
In view (4.19) we have

$\overline{P_{5}(x, s)}=\overline{P_{5}(0, s)} e^{-s x} e^{-\int_{0}^{x} \mu_{5} d u}$

In view of (4.30) the Second integral becomes as the following

$$
\begin{aligned}
I I & =\int_{0}^{\infty} \mu_{5}(x) \overline{P_{5}(0, s)} e^{-s x} e^{-\int_{0}^{x} \mu_{5} d u} d x=\overline{P_{5}(0, s)} \int_{0}^{\infty} \mu_{5}(x) e^{-s x} e^{-\int_{0}^{x} \mu_{5} d u} d x \\
& =\overline{P_{5}(0, s)} \overline{q_{5}(s)},
\end{aligned}
$$

where

$\overline{q_{5}(s)}=\int_{0}^{\infty} \mu_{5}(x) e^{-s x} e^{-\int_{0}^{x} \mu_{5} d u} d x$

and by using the boundary condition (4.23), the above integrals becomes as the following

$$
I I=w \overline{P_{4}(s)} \overline{q_{5}(s)} .
$$

From (4.18), we have

$\overline{P_{4}(s)}=\left[\frac{\lambda_{p}}{s+w}+\frac{\lambda_{p a} \lambda_{a}}{(s+w)\left(s+B_{1}\right)}+\frac{\lambda_{p b} \lambda_{b}}{(s+w)\left(s+B_{2}\right)}\right] \overline{P_{0}(s)}$.

From (4.31) we obtain

$I I=w\left[\frac{\lambda_{p}}{s+w}+\frac{\lambda_{p a} \lambda_{a}}{(s+w)\left(s+B_{1}\right)}+\frac{\lambda_{p b} \lambda_{b}}{(s+w)\left(s+B_{2}\right)}\right] \overline{P_{0}(s)} \overline{q_{5}(s)}$.

let the final integral of equation (4.14) is denoted by

$I I I=\int_{0}^{\infty} \mu_{6}(x) \overline{P_{6}(x, s)} d x$

From equation (4.30), we get

$\overline{P_{6}(x, s)}=\overline{P_{6}(0, s)} e^{-s x} e^{-\int_{0}^{x} \mu_{6} d x}$,

By using (4.34) integral III becomes as the following

$I I I=\overline{P_{6}(0, s)} \overline{q_{6}(s)}$

$\overline{P_{6}(0, s)}=\left[\lambda_{c c}+\frac{\lambda_{c c a} \lambda_{a}}{s+B_{1}}+\frac{\lambda_{c c b} \lambda_{b}}{s+B_{2}}\right] \overline{P_{0}(s)}$.

$I I I=\left[\lambda_{c c}+\frac{\lambda_{c c a} \lambda_{a}}{s+B_{1}}+\frac{\lambda_{c c b} \lambda_{b}}{s+B_{2}}\right] \overline{P_{0}(s)} \overline{q_{6}(s)}$.

$I V=\theta_{1} \overline{P_{7}(s)}=\theta_{1} \frac{\theta_{2}}{s+\theta_{1}} \overline{P_{0}(s)}$.

From (4.29), (4.33), (4.37), (4.40) and (4.14) we get

$\overline{P_{0}(s)}=\frac{1}{G(s)}$

where: 
$G(s)=s+\lambda_{c c}+\lambda_{a}+\theta_{2}+\lambda_{b}+\lambda_{P}-\lambda_{a} \lambda_{b}\left(\frac{1}{s+B_{1}}+\frac{1}{s+B_{2}}\right) \overline{q_{3}(s)}-\frac{w}{(s+w)}\left(\lambda_{P}+\frac{\lambda_{a} \lambda_{P a}}{s+B_{1}}+\frac{\lambda_{b} \lambda_{P b}}{s+B_{2}}\right) \overline{q_{5}(s)}-$
$\left(\lambda_{c c}+\frac{\lambda_{a} \lambda_{c c a}}{s+B_{1}}+\frac{\lambda_{b} \lambda_{c c b}}{s+B_{2}}\right) \overline{q_{6}(s)}-\frac{\theta_{2}}{s+\theta_{1}}$.

From (4.30), the marginal of $s$ by integration with respect to $x$ :

$\overline{P_{5}(s)}=\overline{P_{5}(0, s)} \int_{0}^{\infty} e^{-s x} e^{-\int_{0}^{x} \mu_{5} d x} d x$

Integrating the above equation by parts and using(4.23) and (4.32) we have

$\overline{P_{5}(s)}=w\left[\frac{\lambda_{p}}{s+w}+\frac{\lambda_{p a} \lambda_{a}}{(s+w)\left(s+B_{1}\right)}+\frac{\lambda_{p b} \lambda_{b}}{(s+w)\left(s+B_{2}\right)}\right] \overline{P_{0}(s)}\left[\frac{1-\overline{q_{5}(s)}}{s}\right]$.

From (4.34), the marginal of $s$ by integration with respect to $x$ :

$\overline{P_{6}(s)}=\overline{P_{6}(0, s)} \int_{0}^{\infty} e^{-s x} e^{-\int_{0}^{x} \mu_{6} d x} d x$

Integrating the above equation by parts and using(4.34) we have $u$

$\overline{P_{6}(s)}=\left[\lambda_{c c}+\frac{\lambda_{a} \lambda_{c c a}}{s+B_{1}}+\frac{\lambda_{b} \lambda_{c c b}}{s+B_{2}}+\right] \overline{P_{0}(s)} \frac{1-\overline{q_{6}(s)}}{s}$

The Laplace transform of the probabilities that the system is in up and down state at any

Time $t$ is given by the following equations:

$\overline{P_{u p}(s)}=\overline{P_{0}(s)}+\overline{P_{1}(s)}+\overline{P_{2}(s)}+\overline{P_{4}(s)}+\overline{P_{7}(s)}$

$\overline{P_{\text {down }}(s)}=\overline{P_{3}(s)}+\overline{P_{5}(s)}+\overline{P_{6}(s)}$

\section{Availability of the system in Case with Preventive Maintenance}

Availability is defined as the probability that a system is functioning properly at any random timet, Chopar \& Ram (2017). Availability is a performance criterion for the repairable system and is closely associated with the concepts of reliability and maintainability. Availability refers to failure-free operation of the system at a given instant of time, whereas reliability refers to failure-free operation of the system during an interval.

$$
A(t)=\sum u p \text { stat }
$$

We have measured the availability of the system in following particular cases:

\subsection{Availability of the system in comprehensive state.}

Let: $\lambda_{a}=0.50, \lambda_{b}=0.40, \lambda_{P}=0.25, \lambda_{P a}=0.20, \lambda_{P b}=0.10, \lambda_{c c}=0.25, \lambda_{c c a}=0.20, \quad \lambda_{c c b}=0.10$, $w=0.30, \mu_{3}(x)=1, \quad \mu_{5}(x)=1, \quad \mu_{6}(x)=1, \quad \theta_{1}=0.2, \quad \theta_{2}=0.3$

By inverse Laplace transform of equation (4.43), we get the availability of the system as:

$$
\begin{aligned}
A(t)= & 0.2952767384907252 e^{-1.6345838095948422 t} \operatorname{Cos}(0.44449509588338 t)+ \\
& 0.45217744681454647 e^{-1.6345838095948422 t} \operatorname{Sin}(0.44449509588338 t)- \\
& 0.02545788080007272 e^{-0.22240267261787078 t}-0.05418576883183501 e^{-0.4596306386006044 t}- \\
& 0.0014644492667517106 e^{-0.7487990695918408 t}+0.7858313604079344
\end{aligned}
$$




\subsection{Availability of the system without common cause failure.}

Let: $\lambda_{a}=0.50, \lambda_{b}=0.40, \lambda_{P}=0.25, \lambda_{P a}=0.20, \lambda_{P b}=0.10, \quad \lambda_{c c}=0, \quad \lambda_{c c a}=0$, $\lambda_{c c b}=0, w=0.30, \quad \mu_{3}(x)=1, \mu_{5}(x)=1, \mu_{6}(x)=1, \theta_{1}=0.2, \theta_{2}=0.3$

In equation (4.43), and after that taking inverse Laplace transform, we obtain:

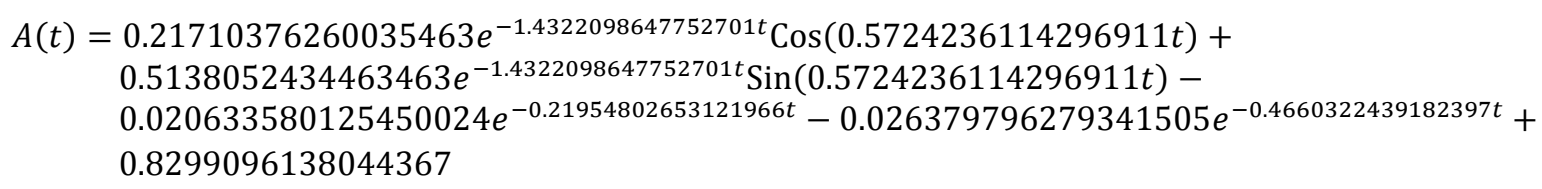

\subsection{Availability of the system without partial failure.}

Let: $\lambda_{a}=0, \lambda_{b}=0, \lambda_{P}=0, \lambda_{P a}=0.20, \lambda_{P b}=0.10, \lambda_{c c}=0.25, \lambda_{c c a}=0.20$,

$\lambda_{c c b}=0.10, w=0.30, \mu_{3}(x)=1, \mu_{5}(x)=1, \quad \mu_{6}(x)=1, \quad \theta_{1}=0.2 ， \theta_{2}=0.3$

Then taking inverse Laplace transform equation(4.43), we attain the availability of the system as follows:

$$
\begin{aligned}
A(t)=0.0011052608350878845 e^{-0.5455464763767969 t}-0.06979003200124803 e^{-0.24144470319751457 t} & \\
+ & 0.7754164195796075 e^{0.01502015007704967 t} \\
& +0.29326835158 e^{-1.4890144852513691 t} \operatorname{Cos}[0.4571489812273525 t] \\
& +0.34734079413 e^{-1.4890144852513691 t} \operatorname{Sin}[0.4571489812273525 t]
\end{aligned}
$$

Information that pertains to the availability of the system with respect to time, when all the failure rates have some fixed values, is furnished in this Table 1 and Figure 5 . An inspection of Figure 5 reveals that the availability of the system decreases sharply during the initial stage but later on stabilizes with the increment of time:

\begin{tabular}{|c|c|c|c|c||c|c||}
\hline \hline \multicolumn{2}{|c||}{ Availability } \\
\hline \multirow{2}{*}{ Time } & \multicolumn{2}{|c|}{ Comprehensive States } & \multicolumn{2}{c||}{$\begin{array}{c}\text { Without common cause } \\
\text { failure States }\end{array}$} & \multicolumn{2}{c||}{ without partial failure } \\
\cline { 2 - 7 } & $\begin{array}{c}\text { With } \\
\text { preventive } \\
\text { maintenance }\end{array}$ & $\begin{array}{c}\text { Without } \\
\text { preventive } \\
\text { maintenance }\end{array}$ & $\begin{array}{c}\text { With } \\
\text { preventive } \\
\text { maintenance }\end{array}$ & $\begin{array}{c}\text { Without } \\
\text { preventive } \\
\text { maintenance }\end{array}$ & $\begin{array}{c}\text { With } \\
\text { preventive } \\
\text { maintenance }\end{array}$ & $\begin{array}{c}\text { Without } \\
\text { preventive } \\
\text { maintenance }\end{array}$ \\
\hline 0 & 1 & 1 & 1 & 1 & 1 & 1 \\
\hline 1 & 0.820453 & 0.801619715 & 0.906823 & 0.898519305 & 0.826925 & 0.797011447 \\
\hline 2 & 0.768009 & 0.730375593 & 0.838014 & 0.813289374 & 0.779487 & 0.713192134 \\
\hline 3 & 0.762741 & 0.716108276 & 0.819202 & 0.784577324 & 0.782123 & 0.689970969 \\
\hline 4 & 0.767234 & 0.716071353 & 0.818038 & 0.779075731 & 0.797665 & 0.685301182 \\
\hline 5 & 0.772032 & 0.717945333 & 0.820408 & 0.779289632 & 0.815137 & 0.684768991 \\
\hline 6 & 0.775671 & 0.719417446 & 0.822706 & 0.780107744 & 0.832175 & 0.684837691 \\
\hline
\end{tabular}




\begin{tabular}{|c|c|c|c|c|c|c|}
\hline 7 & 0.778283 & 0.720371805 & 0.824438 & 0.780651120 & 0.848524 & 0.684901381 \\
\hline 8 & 0.780159 & 0.720975557 & 0.825707 & 0.780955844 & 0.864318 & 0.684924494 \\
\hline 9 & 0.781524 & 0.721359904 & 0.82665 & 0.781129849 & 0.879716 & 0.684930404 \\
\hline 10 & 0.78253 & 0.721606263 & 0.827363 & 0.781234788 & 0.894851 & 0.684931497 \\
\hline 11 & 0.783281 & 0.72176471 & 0.827909 & 0.781300445 & 0.909824 & 0.684931565 \\
\hline 12 & 0.783848 & 0.721866742 & 0.828331 & 0.781342127 & 0.924718 & 0.684931534 \\
\hline 13 & 0.78428 & 0.72193247 & 0.828659 & 0.781368668 & 0.939596 & 0.684931515 \\
\hline 14 & 0.784613 & 0.721974815 & 0.828917 & 0.781385558 & 0.954509 & 0.684931509 \\
\hline 15 & 0.784871 & 0.722002098 & 0.829119 & 0.781396295 & 0.969499 & 0.684931507 \\
\hline
\end{tabular}

Table 1: Availability of the system

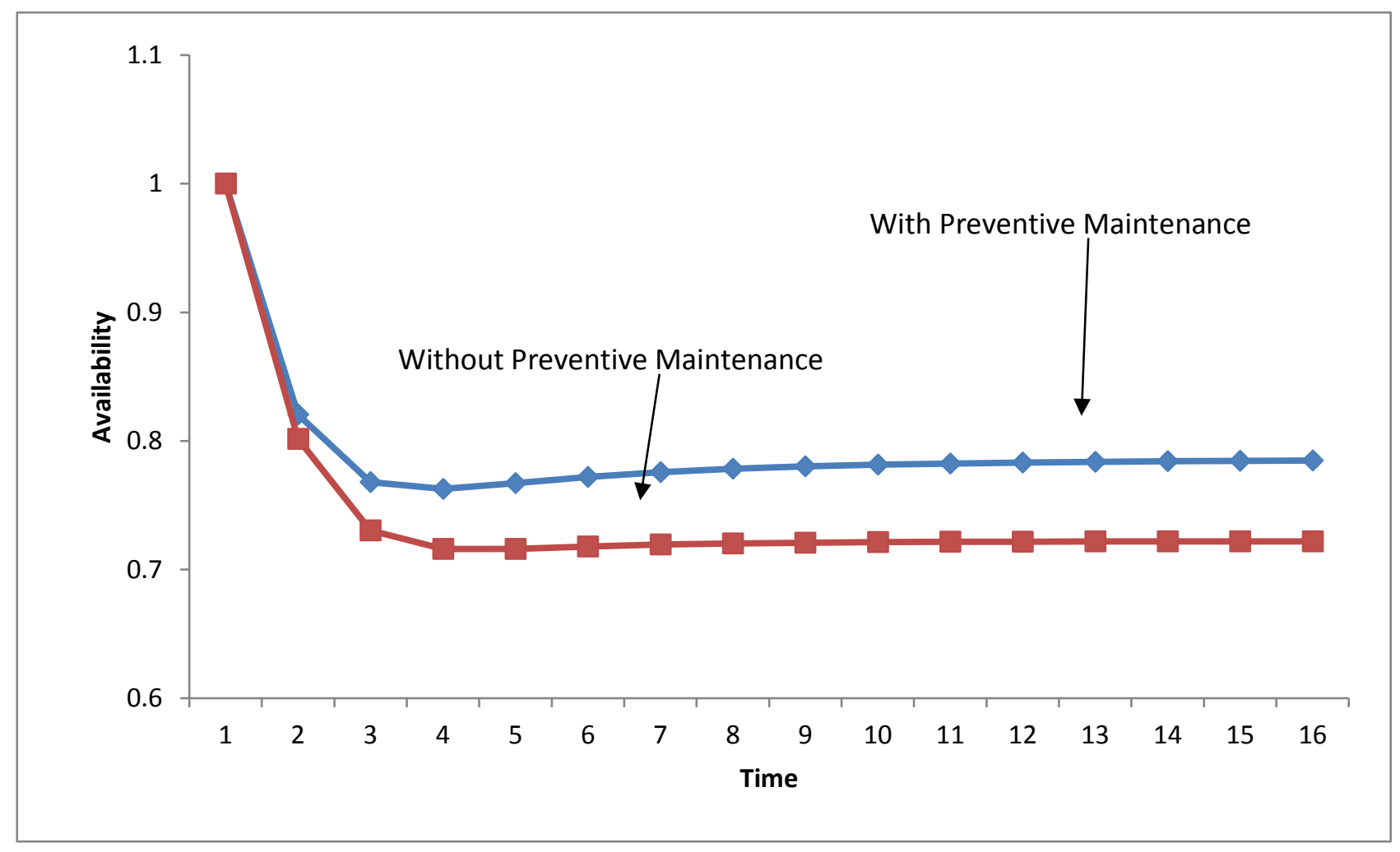

Figure 2: Comparing Availability in comprehensive states in both cases (with and without preventive maintenance) 


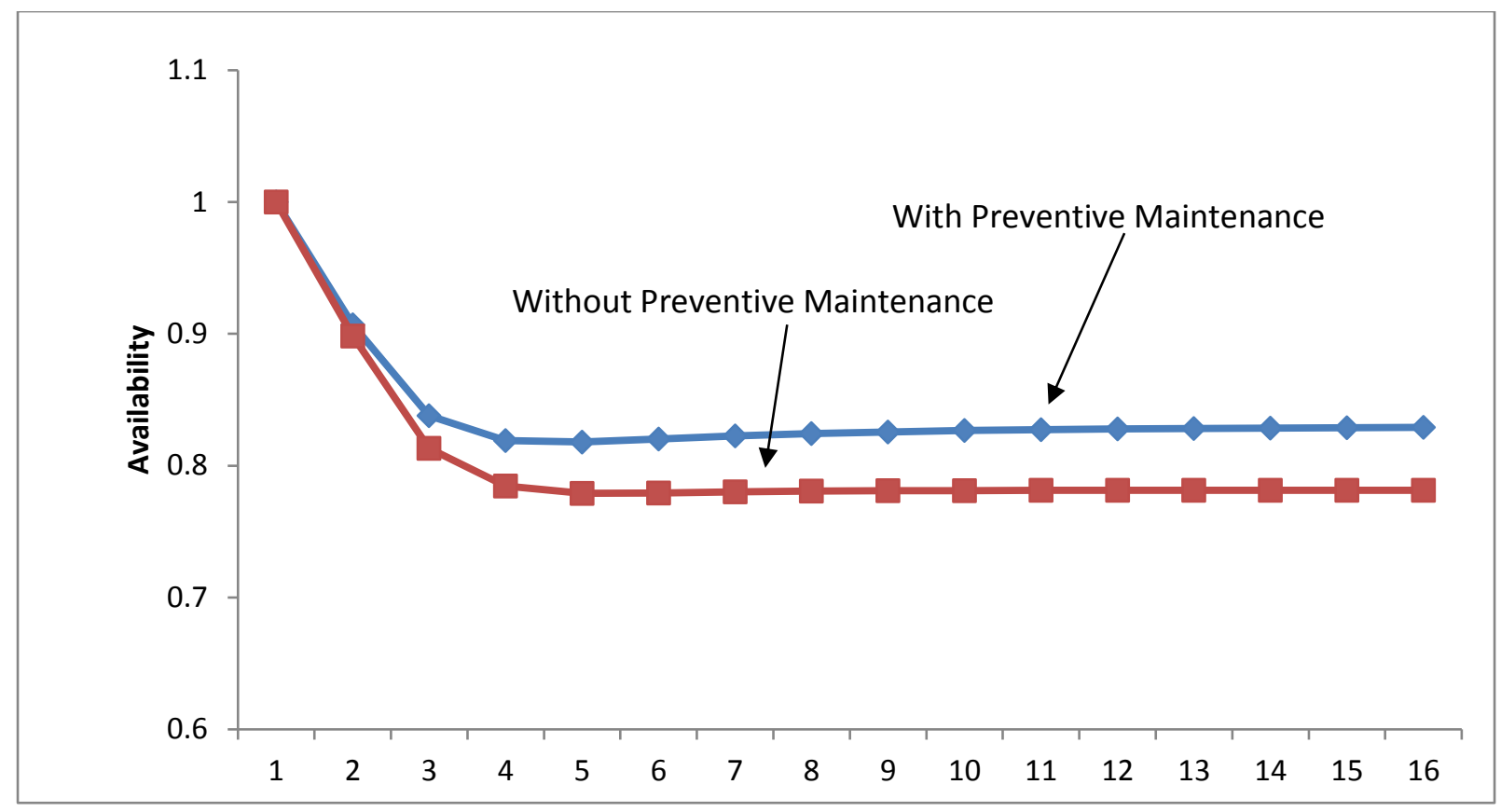

Figure 3: Comparing Availability without common cause failure in both cases (with and without preventive maintenance)

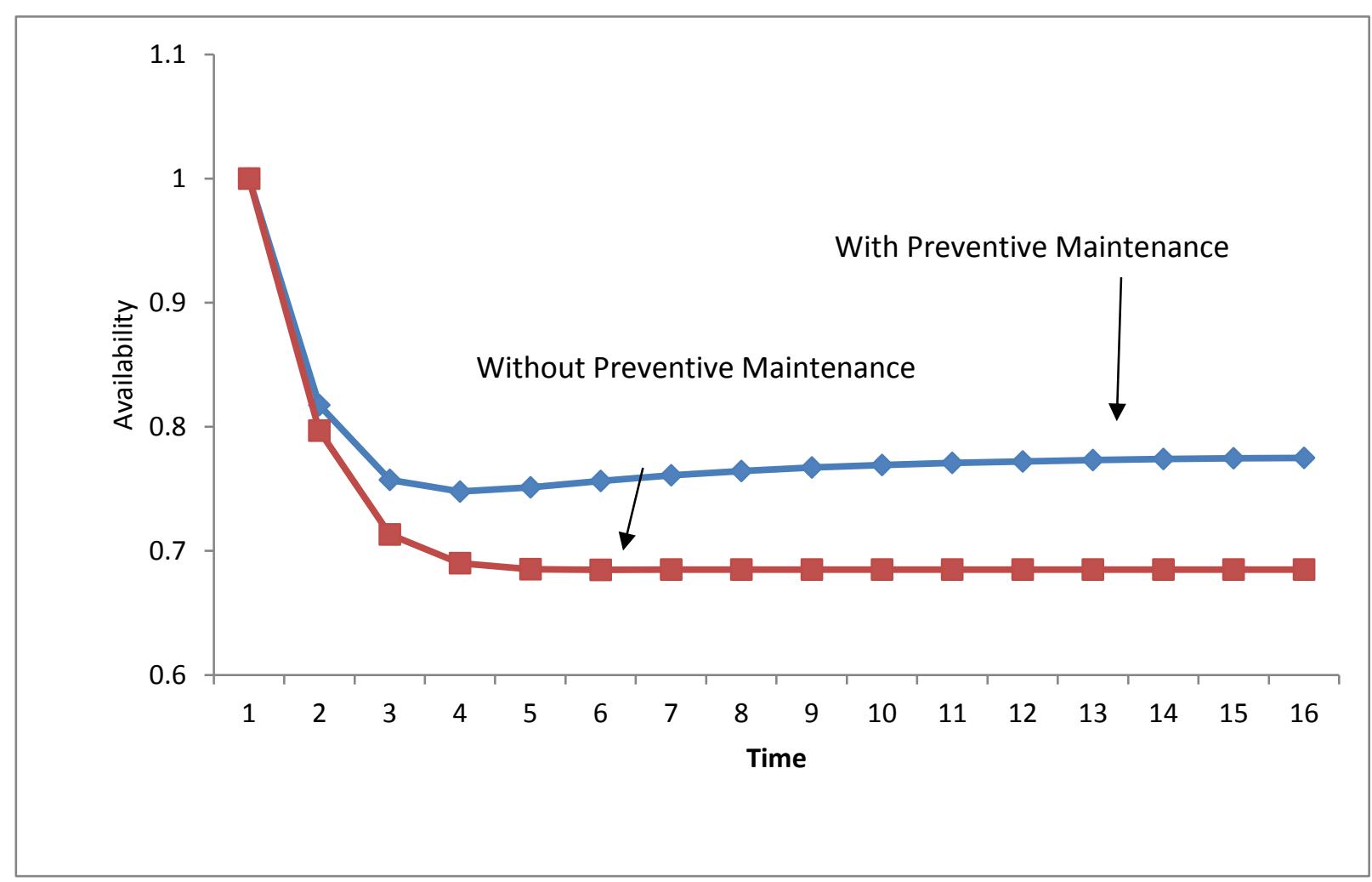

Figure 4: Comparing Availability without partial failure in both cases (with and without preventive maintenance) 


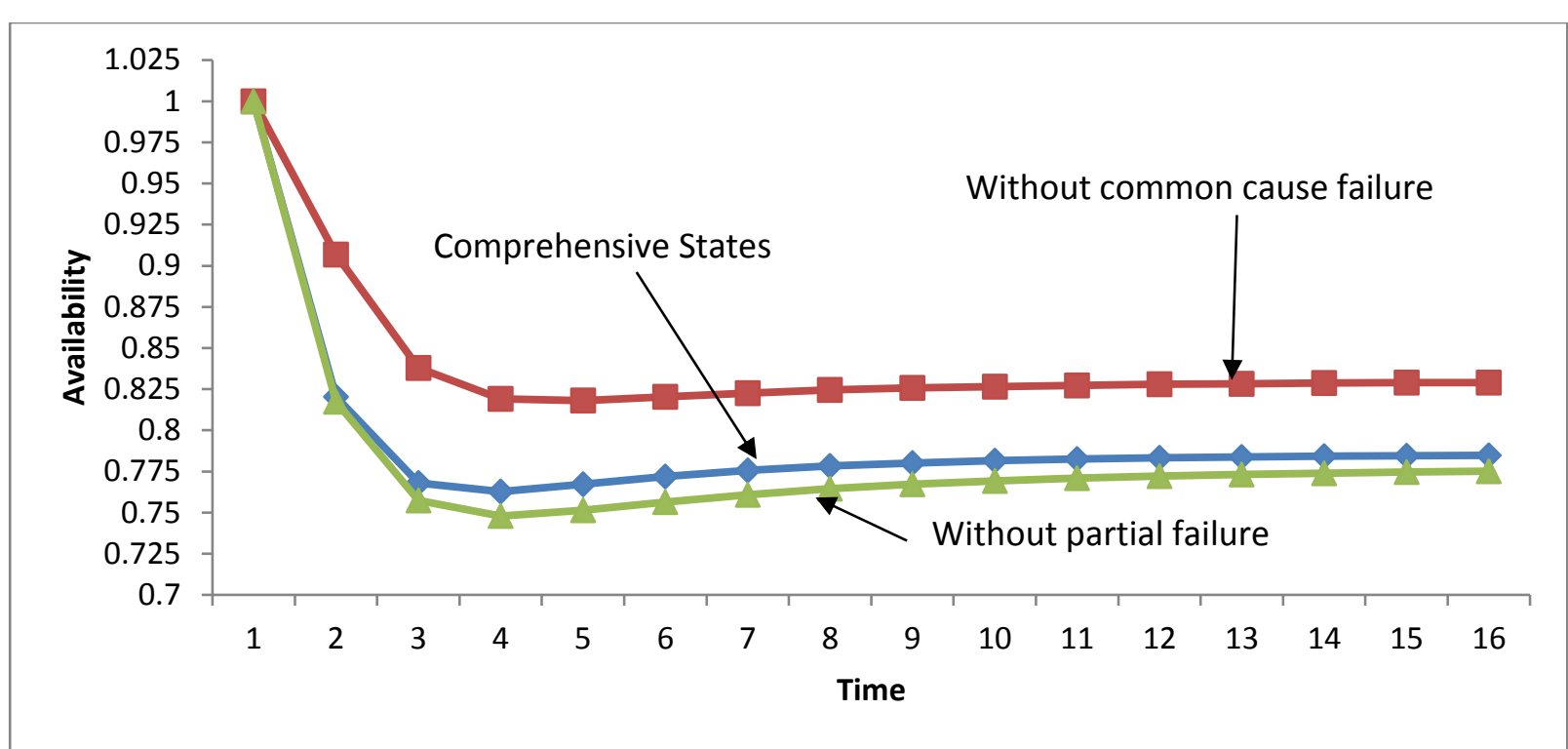

Figure 5: Comparing Availability for system with preventive maintenance in cases Comprehensive states, without common cause failure and without partial failure.

The availability of the system with preventive maintenance stabilizes to $0.78,0.83$ and 0.97 in a long run when the system is free from common cause failure, is in comprehensive state and is without partial failure, respectively. It is very clear from Figure 5 that the availability of the system in comprehensive state is slightly lower than the availability of the system when there is no common cause failure. It can also be seen that the availability of the system is minimum when the system is without partial failure and is maximum when the system is free from common cause failure.

\section{Reliability of the system in Case with Preventive Maintenance}

A sudden failure of any system may results in health hazard, accident or interruption in the continuity of service. Uninterrupted service and hazard-free operation of complex systems like software systems, satellite control systems, nuclear weapons control systems, power supply systems and telecommunication systems have necessitated the study of reliability analysis. Reliability is the probability of a device performing its purpose adequately for the period intended under the given operating conditions. It should be observed that the abovestated definition brings into focus four significant factors, namely, probability, adequate performance, intended time and operating conditions. These four elements play a central role in determining the reliability of an item. The reliability of the present system is obtained by setting all the repair rates to 0 in Equation (3.43), and followed by the inverse Laplace transform. The reliability of the system with respect to different cases is discussed as follows

\subsection{Reliability of the system in comprehensive state.}

Putting all repair rates to 0 in Equation (4.43) and substituting $\lambda_{a}=0.50, \lambda_{b}=0.40$,

$\lambda_{P}=0.25, \lambda_{P a}=0.20, \quad \lambda_{P b}=0.10, \quad \lambda_{c c}=0.25, \lambda_{c c a}=0.20, \lambda_{c c b}=0.10, w=0.30, \quad \theta_{1}=0.2, \quad \theta_{2}=0.3$ and then taking inverse Laplace transform, we get the reliability of the system as follows:

$$
\begin{aligned}
R(t)= & -0.18080285396466123 e^{-1.7389866919029748 t}+0.299999999999989 e^{-0.8 t}+ \\
& 0.2678571428571529 e^{-0.7 t}+0.2750000000000017 e^{-0.3 t}+0.33794571110751825 e^{-0.16101330809702502 t}
\end{aligned}
$$

\subsection{Reliability of the system without common cause failure.}

Setting all the repair rates to 0 in Equation (4.43) and considering $\lambda_{a}=0.50, \lambda_{b}=0.40$, 
$\lambda_{P}=0.25, \lambda_{P a}=0.20, \lambda_{P b}=0.10, \quad \lambda_{c c}=\mathbf{0}, \lambda_{c c a}=\mathbf{0}, \lambda_{c c b}=\mathbf{0}, w=0.30$,

$\theta_{1}=0.2, \quad \theta_{2}=0.3$ and then taking inverse Laplace transform, we get:

$$
\begin{aligned}
R(t)= & -0.30315851932454924 \mathrm{e}^{-1.4962860791048778 t}+0.4333333333333337 \mathrm{e}^{-0.6 t}+ \\
& 0.40952380952380885 \mathrm{e}^{-0.3 t}+0.4603013764674067 \mathrm{e}^{-0.1537139208951224 t}
\end{aligned}
$$

\subsection{Reliability of the system without partial failure.}

Assuming all the repair rates as 0 in Equation (4.43) and substituting $\lambda_{a}=0, \lambda_{b}=0$, $\lambda_{P}=0, \lambda_{P a}=0.20, \lambda_{P b}=0.10, \quad \lambda_{c c}=0.25, \lambda_{c c a}=0.20, \lambda_{c c b}=0.10, w=0.30$, $\theta_{1}=0.2, \quad \theta_{2}=0.3$ and then taking inverse Laplace transform, we obtain:

$R(t)=-0.22745249871463738 e^{-1.49629 t}+0.9 e^{-0.6 t}+0.3274524987146374 e^{-0.153714 t}$

Table 2 and corresponding Figure 9 yield information about the reliability with preventive maintenance of the considered system. It can be concluded that the reliability of the system decreases with the passage of time. Figure 9 depicts that the system has the highest reliability with preventive maintenance when there is no common cause failure and has the lowest reliability when it is without partial failure. It is interesting to find that the reliability of the assumed system is more in the comprehensive state as compared to its reliability when it is free from partial failure.

\begin{tabular}{|c|c|c|c|c|c|c|}
\hline \hline \multirow{2}{*}{ Time } & \multicolumn{2}{|c|}{ Reliability } \\
\hline \hline \multirow{2}{*}{ Time } & \multicolumn{2}{|c|}{$\begin{array}{c}\text { Without common cause } \\
\text { failure States }\end{array}$} & \multicolumn{2}{|c|}{ without partial failure } \\
\cline { 2 - 7 } & $\begin{array}{c}\text { With } \\
\text { maintentive }\end{array}$ & $\begin{array}{c}\text { Without } \\
\text { preventive } \\
\text { maintenance }\end{array}$ & $\begin{array}{c}\text { With } \\
\text { preventive } \\
\text { maintenance }\end{array}$ & $\begin{array}{c}\text { Without } \\
\text { preventive } \\
\text { maintenance }\end{array}$ & $\begin{array}{c}\text { With } \\
\text { preventive } \\
\text { maintenance }\end{array}$ & $\begin{array}{c}\text { Without } \\
\text { preventive } \\
\text { maintenance }\end{array}$ \\
\hline \hline 0 & 1 & 1 & 1 & 1 & 1 & 1 \\
\hline 1 & 0.727458 & 0.702211452 & 0.86802 & 0.857205606 & 0.723786 & 0.696559279 \\
\hline 2 & 0.516866 & 0.454977035 & 0.678538 & 0.636763128 & 0.500454 & 0.429062173 \\
\hline 3 & 0.379323 & 0.294698457 & 0.524972 & 0.452997509 & 0.352693 & 0.250287321 \\
\hline 4 & 0.288649 & 0.195454865 & 0.410788 & 0.319079942 & 0.258134 & 0.142050937 \\
\hline 5 & 0.225996 & 0.133273829 & 0.326211 & 0.225347140 & 0.196512 & 0.079444342 \\
\hline 6 & 0.18055 & 0.093094622 & 0.262516 & 0.160261503 & 0.154761 & 0.044070228 \\
\hline 7 & 0.146264 & 0.066244774 & 0.213581 & 0.114860915 & 0.125137 & 0.024335152 \\
\hline 8 & 0.119639 & 0.047768694 & 0.175297 & 0.082907972 & 0.103145 & 0.013402561 \\
\hline 9 & 0.0985394 & 0.034761592 & 0.144885 & 0.060201802 & 0.0861629 & 0.007370410 \\
\hline 10 & 0.0815788 & 0.025451716 & 0.120425 & 0.043923896 & 0.0726315 & 0.004049694 \\
\hline 11 & 0.067807 & 0.018710931 & 0.100556 & 0.032167354 & 0.0615941 & 0.002224016 \\
\hline 12 & 0.0565412 & 0.013792076 & 0.0842839 & 0.023625227 & 0.0524401 & 0.001221040 \\
\hline 13 & 0.0472729 & 0.010184023 & 0.0708692 & 0.017389328 & 0.0447608 & 0.000670271 \\
\hline 14 & 0.0396133 & 0.007528389 & 0.0597494 & 0.012820433 & 0.0382694 & 0.000367900 \\
\hline 15 & 0.0332596 & 0.005569371 & 0.0504894 & 0.009463620 & 0.0327542 & 0.000201923 \\
\hline
\end{tabular}




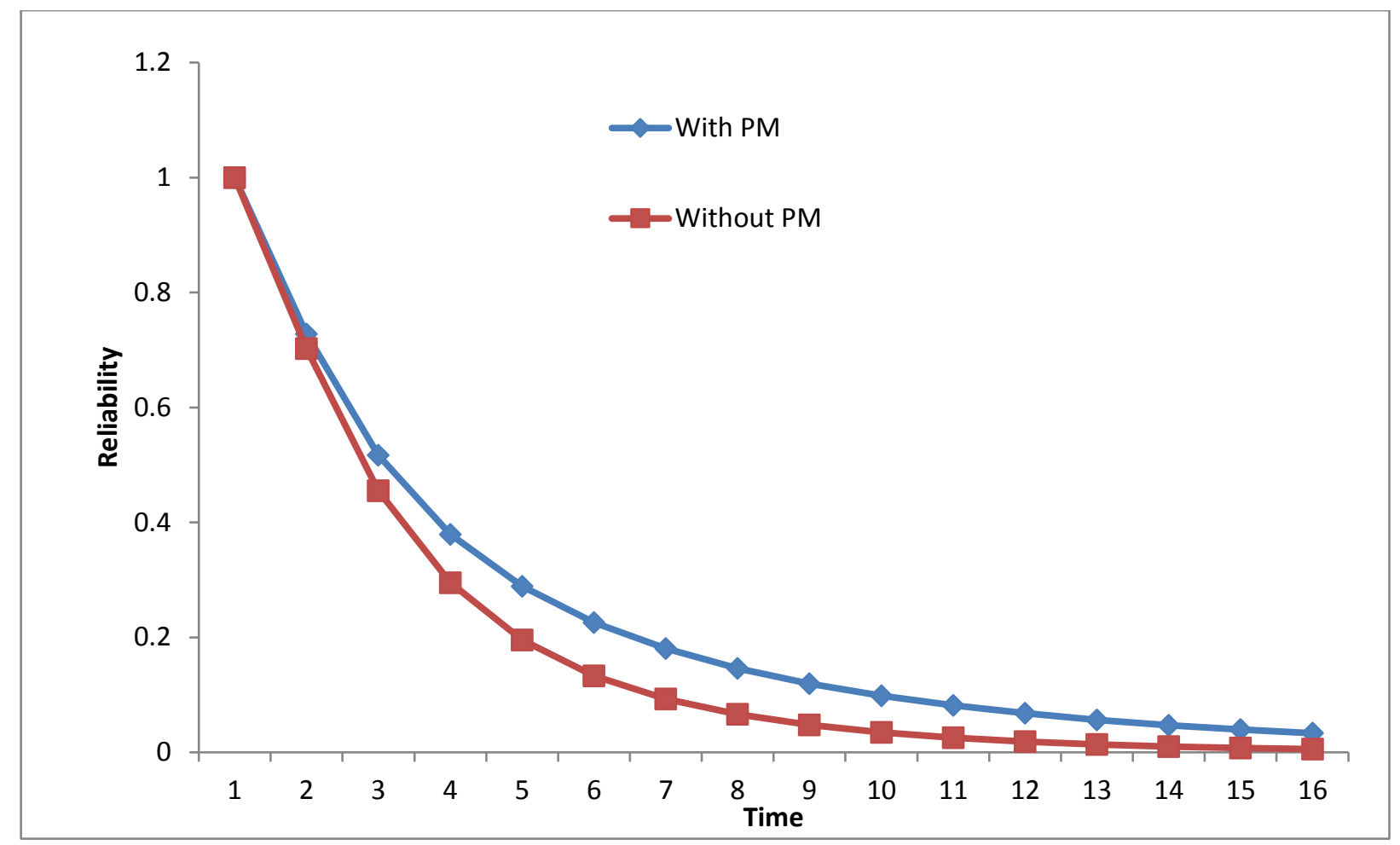

Figure 6: Comparing Reliability in comprehensive states in both cases (with and without preventive maintenance)

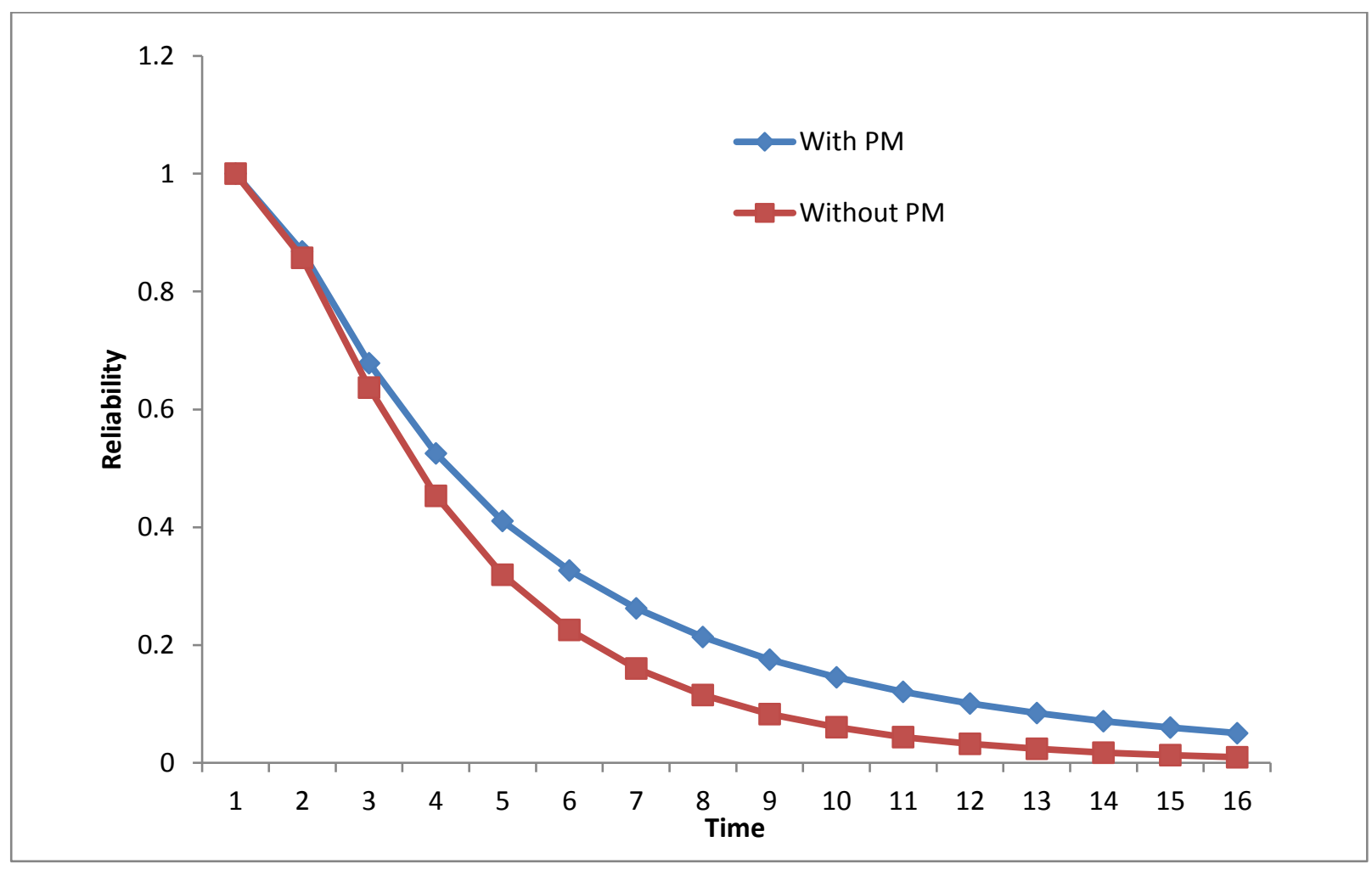

Figure 7: Comparing Reliability without common cause failure in both cases (with and without preventive maintenance) 


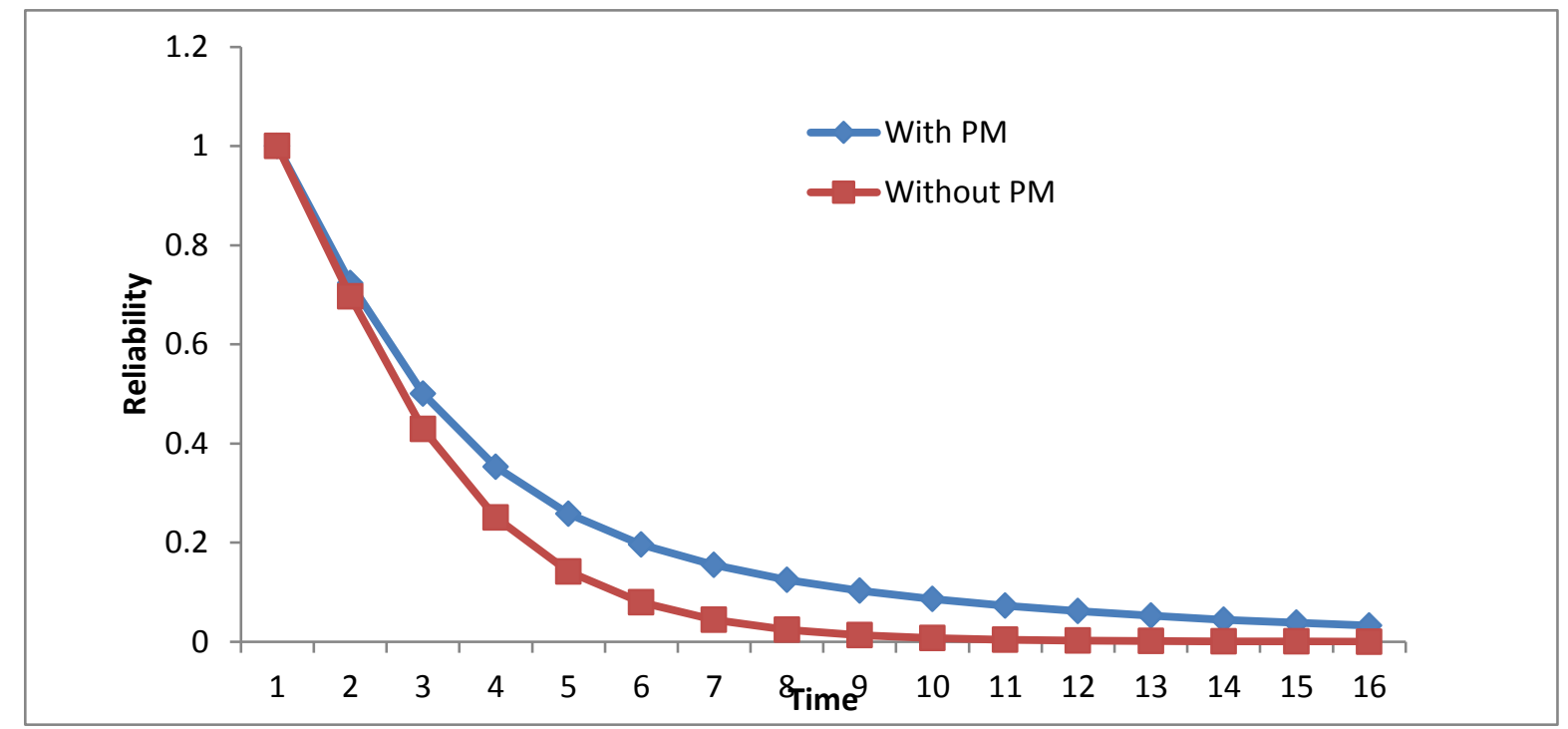

Figure 8: Comparing Reliability without partial failure in both cases (with and without preventive maintenance)

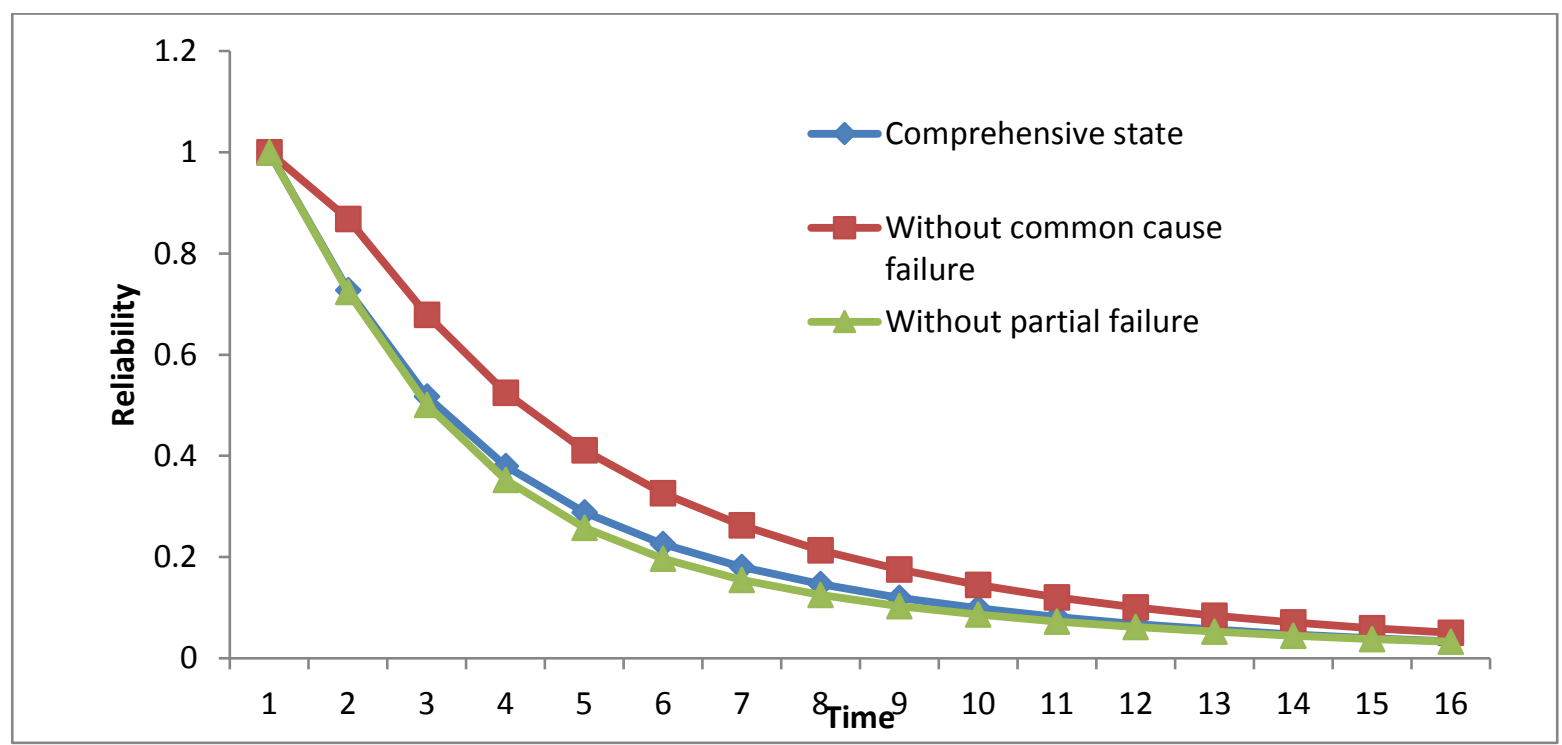

Figure 9: Comparing Reliability for system with preventive maintenance in cases Comprehensive states, without common cause failure and without partial failure.

\section{Mean Time to Failure (MTTF) of the system in Case with Preventive Maintenance:}

A system cannot operate for infinitely long time due to various reasons such as degradation of its components, unfavorable environmental conditions, manufacturing defects, etc. Hence, there is a call for measuring mean life time of the system. MTTF is defined as the mean time for which the system is expected to be operational. It is one of the most widely used parameters of reliability. This metric should be used when failure time distribution is known because the reliability level implied by the MTTF depends on the underlying failure time distribution (Pham, 2007). MTTF is calculated by considering all the repair rates as 0 in Equation (4.43) followed by taking limit as $s$ tends to 0 . 
We have:

$$
\mathrm{MTTF}=\lim _{s \rightarrow 0} \overline{p_{u p}}(s)
$$

we have considered the following cases:

\subsection{MTTF when the system is in the comprehensive state.}

Taking all the repair rates to 0 in Equation (4.43) as s tends to 0 , we get MTTF of the system as:

$M T T F=\left[\frac{1}{\lambda_{c c}+\lambda_{a}+\lambda_{b}+\lambda_{p}}\right]\left[1+\frac{\lambda_{a}}{B_{1}}+\frac{\lambda_{b}}{B_{2}}+\frac{\lambda_{p}}{w}+\frac{\lambda_{a} \lambda_{p a}}{w B_{1}}+\frac{\lambda_{b} \lambda_{p b}}{w B_{2}}+\frac{\theta_{2}}{\theta_{1}}\right]$

Substituting $\lambda_{a}=0.50, \lambda_{b}=0.40, \lambda_{P}=0.25, \lambda_{P a}=0.20, \lambda_{P b}=0.10$,

$\lambda_{c c}=0.25, \lambda_{c c a}=0.20, \lambda_{c c b}=0.10, w=0.30, \theta_{1}=0.2, \theta_{2}=0.3$ and varying failure rates $\lambda_{a}, \lambda_{b}, \lambda_{P}, \lambda_{c c}, \mathrm{w}$, $\lambda_{P a}, \lambda_{P b}, \lambda_{c c a}, \lambda_{c c b}$ one at a time in Equation (7.1) as $0.1,0.2,0.3,0.4,0.5,0.6,0.7,0.8,0.9$, we have studied the effect of change of failure rates on MTTF.

\section{MTTF}

\begin{tabular}{|c|c|c|c|c|c||c|c||c||c||c|}
\hline \hline$\lambda_{a}$ & $\lambda_{b}$ & $\lambda_{P}$ & $\lambda_{c c}$ & $w$ & $\lambda_{P a}$ & $\lambda_{P b}$ & $\lambda_{c c a}$ & $\lambda_{c c b}$ & $\theta_{1}$ \\
\hline 5.31944 & 4.71861 & 3.70952 & 4.10952 & 5.72704 & 3.60544 & 3.66922 & 3.77551 & 3.66922 & 4.74065 & 2.95493 \\
\hline 4.62121 & 4.25265 & 3.68166 & 3.80511 & 4.18367 & 3.66922 & 3.72024 & 3.66922 & 3.60119 & 3.66922 & 3.31207 \\
\hline 4.1875 & 3.91941 & 3.65764 & 3.54269 & 3.66922 & 3.71882 & 3.75992 & 3.58655 & 3.54828 & 3.31207 & 3.66922 \\
\hline 3.88889 & 3.66922 & 3.63671 & 3.31413 & 3.41199 & 3.7585 & 3.79167 & 3.52041 & 3.50595 & 3.1335 & 4.02636 \\
\hline 3.66922 & 3.47443 & 3.61833 & 3.11328 & 3.25765 & 3.79097 & 3.81764 & 3.4663 & 3.47132 & 3.02636 & 4.3835 \\
\hline 3.5 & 3.31845 & 3.60204 & 2.93537 & 3.15476 & 3.81803 & 3.83929 & 3.4212 & 3.44246 & 2.95493 & 4.74065 \\
\hline 3.36516 & 3.19073 & 3.58752 & 2.77671 & 3.08127 & 3.84092 & 3.8576 & 3.38305 & 3.41804 & 2.90391 & 5.09779 \\
\hline 3.2549 & 3.08422 & 3.57448 & 2.63431 & 3.02615 & 3.86054 & 3.8733 & 3.35034 & 3.39711 & 2.86565 & 5.45493 \\
\hline 3.16288 & 2.99402 & 3.56272 & 2.50581 & 2.98328 & 3.87755 & 3.8869 & 3.322 & 3.37897 & 2.83588 & 5.81207 \\
\hline
\end{tabular}

\subsection{MTTF when the system is without common cause failure.}

Setting $\lambda_{c c}=0, \lambda_{c c a}=0, \lambda_{c c b}=0$ in Equation (7.1) we obtain MTTF of the System as:

$M T T F=\left[\frac{1}{\lambda_{a}+\lambda_{b}+\lambda_{p}}\right]\left[1+\frac{\lambda_{a}}{\lambda_{b}+\lambda_{p a}}+\frac{\lambda_{b}}{\lambda_{a}+\lambda_{p b}}+\frac{\lambda_{p}}{w}+\frac{\lambda_{a} \lambda_{p a}}{w\left(\lambda_{a}+\lambda_{p a}\right)}+\frac{\lambda_{b} \lambda_{p b}}{w\left(\lambda_{a}+\lambda_{p b}\right)}+\frac{\theta_{2}}{\theta_{1}}\right]$

Assuming $\lambda_{a}=0.50, \lambda_{b}=0.40, \lambda_{P}=0.25, \lambda_{P a}=0.20, \quad \lambda_{P b}=0.10$ and $w=0.30, \theta_{1}=0.2, \theta_{2}=0.3$ and varying failure rates $\lambda_{a}, \lambda_{b}, \lambda_{P}, w, \lambda_{P a}, \lambda_{P b}$ one by one in Equation (7.2) as $0.1,0.2,0.3,0.4,0.5,0.6,0.7,0.8$, 0.9 , we have explored the effect of change of failure rates on MTTF. 


\begin{tabular}{|c|c|c|c|c|c|c|c|}
\hline \multicolumn{7}{|c|}{ MTTF } \\
\hline$\lambda_{a}$ & $\lambda_{b}$ & $\lambda_{P}$ & $w$ & $\lambda_{P a}$ & $\lambda_{P b}$ & $\theta_{1}$ & $\theta_{2}$ \\
\hline \hline 8.37037 & 7.45098 & 5.94444 & 7.92271 & 5.20359 & 5.12077 & 6.42512 & 4.25121 \\
\hline 6.66667 & 6.16959 & 5.32828 & 5.82126 & 5.12077 & 5.17598 & 5.12077 & 4.68599 \\
\hline 5.78947 & 5.39683 & 4.9537 & 5.12077 & 5.08313 & 5.21739 & 4.68599 & 5.12077 \\
\hline 5.24868 & 4.87923 & 4.70085 & 4.77053 & 5.06503 & 5.2496 & 4.4686 & 5.55556 \\
\hline 4.87923 & 4.50794 & 4.51814 & 4.56039 & 5.05636 & 5.27536 & 4.33816 & 5.99034 \\
\hline 4.60952 & 4.2284 & 4.37963 & 4.42029 & 5.05257 & 5.29644 & 4.25121 & 6.42512 \\
\hline 4.40329 & 4.01022 & 4.27083 & 4.32022 & 5.05143 & 5.31401 & 4.1891 & 6.8599 \\
\hline 4.2401 & 3.83513 & 4.18301 & 4.24517 & 5.05176 & 5.32887 & 4.14251 & 7.29469 \\
\hline 4.10753 & 3.69146 & 4.11055 & 4.1868 & 5.05291 & 5.34161 & 4.10628 & 7.72947 \\
\hline
\end{tabular}

\subsection{MTTF when the system is without partial failure.}

In this particular case we have assumed that the system is free from any partial failure and hence Equation (7.2) reduces to:

MTTF $=\left[\frac{1}{\lambda_{c c}+\lambda_{a}+\lambda_{p}}\right]\left[1+\frac{\lambda_{a}}{\lambda_{b}+\lambda_{c c a}}+\frac{\lambda_{b}}{\lambda_{a}+\lambda_{c c b}}+\frac{\theta_{2}}{\theta_{1}}\right]$

Putting $\lambda_{a}=0.5, \lambda_{b}=0.4, \quad \lambda_{P}=\mathbf{0}, \lambda_{P a}=\mathbf{0}, \quad \lambda_{P b}=\mathbf{0}, \quad \lambda_{c c}=0.25$,

$\lambda_{c c a}=0.20, \lambda_{c c b}=0.10, w=0.30, \theta_{1}=0.2, \theta_{2}=0.3$ and varying failure rates $\lambda_{a}, \lambda_{b}, \lambda_{c c} \lambda_{c c a}$

$\lambda_{c c b}$ individually in Equation (7.3) as $0.1,0.2,0.3,0.4,0.5,0.6,0.7,0.8,0.9$, we have calculated MTTF.

\begin{tabular}{|c|c|c|c|c|c|c|}
\hline \multicolumn{7}{|c|}{ MTTF } \\
\hline \hline$\lambda_{a}$ & $\lambda_{b}$ & $\lambda_{c c}$ & $\lambda_{c c a}$ & $\lambda_{c c b}$ & $\theta_{1}$ & $\theta_{2}$ \\
\hline 6.22222 & 5.09804 & 4.0 & 3.62319 & 3.47826 & 4.78261 & 2.6087 \\
\hline 4.90196 & 4.29825 & 3.63636 & 3.47826 & 3.39545 & 3.47826 & 3.04348 \\
\hline 4.21053 & 3.80952 & 3.33333 & 3.37474 & 3.33333 & 3.04348 & 3.47826 \\
\hline 3.77778 & 3.47826 & 3.07692 & 3.2971 & 3.28502 & 2.82609 & 3.91304 \\
\hline 3.47826 & 3.2381 & 2.85714 & 3.23671 & 3.24638 & 2.69565 & 4.34783 \\
\hline 3.25714 & 3.05556 & 2.66667 & 3.18841 & 3.21476 & 2.6087 & 4.78261 \\
\hline 3.08642 & 2.91188 & 2.5 & 3.14888 & 3.18841 & 2.54658 & 5.21739 \\
\hline 2.95019 & 2.7957 & 2.35294 & 3.11594 & 3.16611 & 2.5 & 5.65217 \\
\hline 2.83871 & 2.69972 & 2.22222 & 3.08807 & 3.147 & 2.46377 & 6.08696 \\
\hline
\end{tabular}




\section{Conclusion}

In this paper, we have studied a parallel system with two non-identical unit have two types of failure: common cause failure and partial failure, considering that units could go for preventive maintenance (PM). Our study differ from the study of Chopra and Ram (2016) due to that we studied the effect of preventive maintenance (PM) on their system. This study showed that the performance measures of this system with preventive maintenance (PM) was better than those of same system without preventive maintenance (PM). We had shown that Availability, Reliability and mean time to failure (MTTF) of the system with preventive maintenance (PM) were higher than those for system without preventive maintenance (PM). Same results have been achieved as Chopra and Ram (2017) regarding Comprehensive state case, without common cause failure, and without partial failure.

\section{References}

[1] Agnihotri, R.K. and Satsangi, S.K. (1996), "Two-non-identical unit-system with priority based repair and inspection", Microelectronics Reliability, Vol. 36 No. 2, pp. 279-282.

[2] Chander, S. (2005), "Reliability models with priority for operation and repair with arrival time of server", Pure and Applied Mathematika Sciences, Vol. 61 Nos 1-2, pp. 9-22.

[3] Chopra, G., \& Ram, M. (2017), "Stochastic analysis of two non-identical unit parallel system incorporating waiting time. International Journal of Quality \& Reliability Management, Vol. 34 No.6, pp. 817-832.

[4] Dhillon, B.S. and Anuda, O.C. (1993a), "Common cause failure analysis of a non identical unit parallel system with arbitrarily distributed repair times", Microelectronics Reliability, Vol. 33 No. 1, pp. 87-103.

[5] Dhillon, B.S. and Anuda, O.C. (1993b), "Common cause failure analysis of a parallel system with warm standby", Microelectronics Reliability, Vol. 33 No. 9, pp. 1321-1342.

[6] Dhillon, B.S. and Viswanath, H.C. (1991), "Reliability analysis of a nonidentical unit parallel system with common-cause failures", Microelectronics Reliability, Vol. 31 Nos 2-3, pp. 429-441.

[7] Dhillon, B.S. and Yang, N. (1992a), "Reliability and availability analysis of warm standby systems with common cause failures and human errors", Microelectronics Reliability, Vol. 32 No. 4, pp. 561-575.

[8] Dhillon, B.S. and Yang, N. (1992b), "Stochastic analysis of standby systems with common cause failures and human errors", Microelectronics Reliability, Vol. 32 No. 12, pp. 16991712.

[9] El-Said, K. M., \&El-hamid, R. A. (2008), "Comparison of reliability characteristics of two systems with preventive maintenance and different modes", Information and Management sciences, Vol. 19 No. 1, pp. 107-11.

[10] Dhillon, B.S. and Yang, N. (1993), "Availability of a man-machine system with critical and non-critical human error", Microelectronics Reliability, Vol. 33 No. 10, pp. 1511-1521.25

[11] Gupta, P.P. and Agarwal, S.C. (1984), "A parallel redundant complex system with two types of failure under preemptive-repeat repair discipline", Microelectronics Reliability, Vol. 24 No. 3, pp. 395-399.

[12] Gupta, P.P. and Sharma, M.K. (1993), "Reliability and MTTF evaluation of a two duplex-unit standby system with two types of repair", Microelectronics Reliability, Vol. 33 No. 3, pp. 291-295.

[13] Kadyan, M.S., Chander, S. and Grewal, A.S. (2004), "Stochastic analysis of nonidentical units reliability models with priority and different modes of failure", Journal of Decision and Mathematical Sciences, Vol. 9 Nos 1-3, pp. 59-82. 
[14] Kontoleon, J.M. and Kontoleon, N. (1974), "Reliability analysis of a system subjected to partial and catastrophic failures", IEEE Transactions on Reliability, Vol. 23 No. 4, pp. 277-278.

[15] Kumar, P., Bharti, A. and Gupta, A. (2012), "Reliability analysis of a two non identical unit system with repair and replacement having correlated failures and repairs", Journal of Informatics \& Mathematical Sciences, Vol. 4 No. 3, pp. 339-350.

[16] Malik, S.C., Bhardwaj, R.K. and Grewal, A.S. (2010), "Probabilistic analysis of a system of two non-identical parallel units with priority to repair subject to inspection", Journal of Reliability and Statistical Studies, Vol. 3 No. 1 , pp. 1-11.

[17] Mohamed, S. and Sherbeny, E.L. (2013), "Stochastic analysis of a two non identical unit parallel system with different types of failures subject to preventive maintenance and repairs", Mathematical Problems in Engineering, Vol. 2013, Article ID 192545, p. 10. Pham, H. (2007), System Software Reliability, Springer-Verlag, London.

[18] Ram, M. and Kumar, A. (2015), "Performability analysis of a system under1-out-of-2:G scheme with perfect reworking", Journal of the Brazilian Society of Mechanical Sciences and Engineering, Vol. 37 No. 3, pp. 10291038.

[19] Ram, M. and Manglik, M. (2014), "Stochastic behaviour of a Markov model under multi-state failures", International Journal of System Assurance: Engineering and Management, Vol. 5 No. 4, pp. 686-699. 26

[20] Ram, M., Singh, S.B. and Singh, V.V. (2013), "Stochastic analysis of a standby system with waiting repair strategy", IEEE Transactions on Systems, Man and Cybernetics, Vol. 43 No. 3, pp. 698-707.

[21] Singh, V.V. and Gulati, J. (2014), "Availability and cost analysis of a standby complex system with different type of failure, under waiting repair discipline using Gumbel-Hougaard copula distribution", in Sharma, A. et al. (Eds), Proceedings of the International Conference on Issues and Challenges in Intelligent Computing Techniques in Ghaziabad, IEEE, pp. 180-186.

[22] Sridharan, V. and Mohanavadivu, P. (1997), "Reliability and availability analysis for two non identical unit parallel system with conman cause failures and human errors", Microelectronics Reliability, Vol. 37 No. 5, pp. 747- 752.

[23] Osaki, Shunji, and Tatsuyuki Asakura. (1970), "A Two-Unit Standby Redundant System with Repair and Preventive Maintenance", Journal of Applied Probability Vol. 7 No. 3, pp. 641-648.

[24] Osaki, S. (1972), "Reliability analysis of a two-unit standby-redundant system with preventive maintenance", IEEE Transactions on Reliability, Vol. 21 No. 1, pp. 24-29.

[25] Subramanian, R., Natarajan, R., \& Ravichandran, N. (1981), "Complex twounit system with preventive maintenance", Microelectronics Reliability, Vol. 21 No. 4, PP. 559-567.

[26] Sugasawa, Y., Kaji, I., \& Kurihara, M. (1983), "Reliability analysis and preventive maintenance for two-unit standby redundant system with different failure time distributions" Electronics and Communications in Japan (Part I: Communications), Vol. 66 No. 2, pp. 19-26.

[27] Zijlstra, M. (1983), "Optimization of preventive maintenance for two-unit cold standby redundant systems with a general cost structure", Mathematical Methods of Operations Research, Vol. 27 No. 1, pp. 55-63. 